\title{
The diagnosis of pulmonary tuberculosis: a Korean perspective
}

\author{
Yeon Joo Jeong ${ }^{1,2}$, Kyung Soo Lee ${ }^{3}$, Jae-Joon Yim ${ }^{4}$ \\ ${ }^{1}$ Department of Radiology, Pusan National University Hospital, Pusan National University School of Medicine, Busan, Korea \\ ${ }^{2}$ Biomedical Research Institute, Pusan National University Hospital, Pusan National University School of Medicine, Busan, Korea \\ ${ }^{3}$ Department of Radiology and Center for Imaging Science, Samsung Medical Center, Sungkyunkwan University School of \\ Medicine, Seoul, Korea \\ ${ }^{4}$ Division of Pulmonary and Critical Care Medicine, Department of Internal Medicine, Seoul National University College of \\ Medicine, Seoul, Korea
}

Received: April 9, 2017

Revised: May 22, 2017

Accepted: June 2, 2017

Corresponding author:

Jae-Joon Yim

Division of Pulmonary and Critical

Care Medicine, Department of Internal Medicine, Seoul National University College of Medicine, 101 Daehak-ro, Jongno-gu, Seoul 03080, Korea

Tel: +82-2-2072-2228

E-mail: yimjj@snu.ac.kr

\section{ABSTRACT}

Despite global efforts to eliminate tuberculosis, the disease remains of huge global public health problem. Prompt and accurate diagnosis of pulmonary tuberculosis is essential to ensure appropriate therapy. Chest radiography, sputum smear microscopy, mycobacterial culture, and nucleic acid amplification test are essential diagnostic elements, and appropriate use of recently introduced molecular tests and chest computed tomography could improve the accuracy of early diagnosis.

Keywords: Diagnosis; Respiratory tract infections; Tuberculosis

\section{INTRODUCTION}

Tuberculosis (TB) continues to be one of the greatest challenges to global health. In 2015, 10.4 million people were estimated to have developed TB and 1.4 million died of the disease [1]. Of these 10.4 million new cases, $56 \%$ were men, $34 \%$ were women, and $10 \%$ were children. People with human immunodeficiency virus (HIV) accounted for 1.2 million (11\%) of all new TB cases, and although the number of TB associated deaths fell by 22\% between 2000 and 2015, TB remained one of the top 10 causes of death worldwide in 2015.

TB cases of 40,847 were notified in South Korea in 2015, and an annual incidence of TB is 80 per 100,000 of the population, which is much higher than its reported incidences in Japan (17 per 100,000) and the USA $(3.2$ per 100,000) [1]. Among high-income countries, South Korea stands out in terms of its TB burden, despite its astonishing rate of economic development [2].

Prompt diagnosis of TB is essential for community public infection control and for ensuring

This is an Open Access article distributed under the terms of the Creative Commons Attribution Non-Commercial License (http:// creativecommons.org/licenses/ by-nc/4.0/). the appropriate therapy. Mycobacterium tuberculosis culture from specimen is the cornerstone of diagnosis, but mycobacterial culture take considerable time (2 to 6 weeks) to produce results [3]. Acid-fast staining of sputum samples from patients with suspected pulmonary TB, confers cost and time benefits, but its diagnostic sensitivity and specificity are poor [4]. To overcome 
the shortcomings of mycobacterial culture and acid-fast staining, several new molecular and non-molecular assays have been introduced for early TB detection. Chest radiography is useful for the diagnosis of pulmonary TB, but not specific, and chest computed tomography (CT) imaging can also be helpful, especially in inconclusive cases.

In this review, we summarize the current concept of TB manifestation and recent technical advances in the diagnosis, and provide an overview of Korean experience regarding the diagnosis of pulmonary TB.

\section{DEVELOPMENT OF TUBERCULOSIS}

\section{Development of infection}

M. tuberculosis is transmitted from person to person by droplet nuclei containing the organism and spread mainly by coughing. In most cases, the initial infection is clinically silent because host immunity adequately limits further multiplication of bacilli [5].

However, in about $5 \%$ of infected individuals, immunity against tuberculous bacilli is inadequate and clinically active disease develops within 1 year of infection, a condition known as progressive primary TB [6]. Risk factors for progressive primary disease include immunosuppression (especially HIV infection), extremes of age, or a large inoculation of mycobacteria. However, in most infected individuals, TB remains clinically and microbiologically latent for many years. This condition, known as latent TB infection, may be detectable only by a positive tuberculin skin test or interferon $\gamma$ release assay result, or by the presence of radiologically identifiable calcifi-

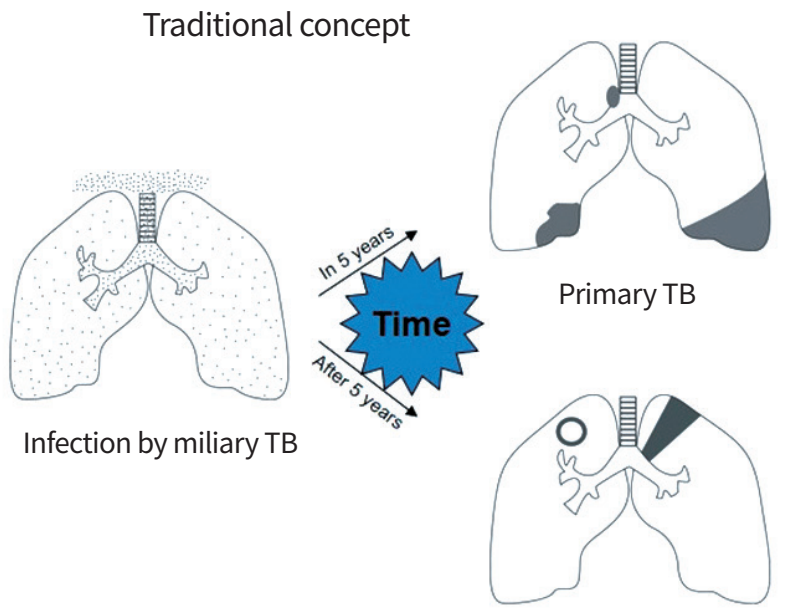

Reactivated TB cation at the site of primary lung infection or in regional lymph nodes [7]. In approximately 5\% of those with a latent TB infection, endogenous reactivation of the infection or reinfection by new strains develops many years after initial infection [8]. Conditions such as suppression of cellular immunity by HIV infection, tumor necrosis factor- $\alpha$ inhibitors, glucocorticoids, organ or hematologic transplantation, and end-stage renal disease increase the risk of postprimary TB development [9].

\section{Current concept of the disease manifestation}

Patients who develop disease after initial exposure are considered to have primary TB, whereas patients who develop disease as a result of reactivation of a remote infection are considered to have postprimary or reactivated TB. Traditionally, it was believed that the clinical, pathologic, and radiologic manifestations of postprimary TB from remote infection were quite different from those of primary TB from recent infection. However, several studies based on DNA fingerprinting suggest that the radiographic features are often similar in patients who have primary disease and those who have postprimary TB [10]. In addition, imaging study revealed that the most common radiographic findings in primary pulmonary TB by recent infection in previously healthy adolescents were consolidation, nodules, and cavities in the upper lung, which were thought to be typical radiographic findings of pulmonary TB reactivation by remote infection [11]. Time from acquisition of infection to development of clinical disease does not reliably predict the radiographic appearance of TB. The only independent predictor of radiographic ap-

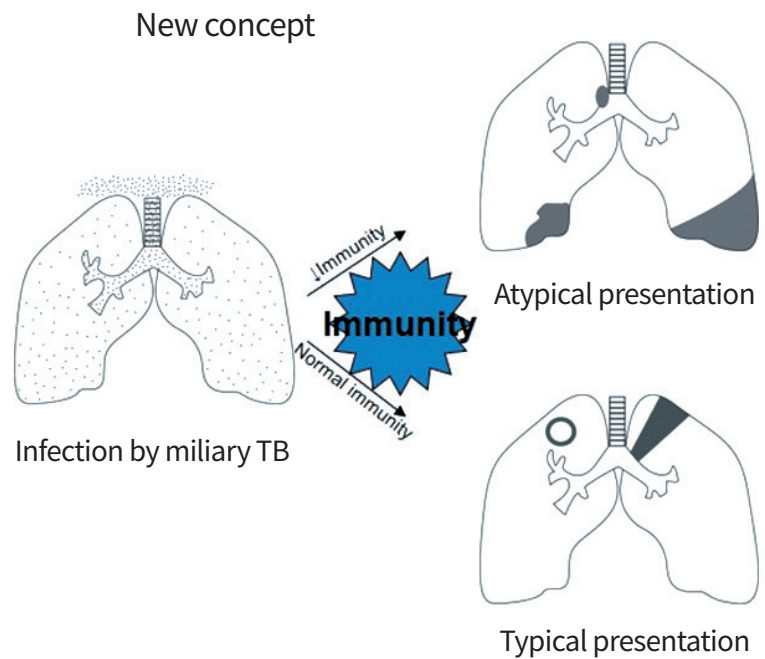

Fig. 1. Traditional and current concepts of tuberculosis (TB) development. The manifestation of pulmonary TB depends on host immunity rather than on time elapsed after initial infection. 
pearance is integrity of host immune response [12]. Immunocompetent patients tend to show parenchymal granulomatous inflammation with slowly progressive nodularity and cavitation, whereas immunocompromised patients have a tendency to develop lymphadenopathy, lower lobe parenchymal consolidation, miliary dissemination, and pleural effusion. These observations suggest that the radiographic presentations of primary and postprimary pulmonary TB depend on host immunity against $M$. tuberculosis rather than time elapsed after initial infection (Fig. 1).

\section{DIAGNOSIS OF TUBERCULOSIS}

\section{Sputum smear microscopy}

Direct sputum smear microscopy and mycobacterial culture are the most widely used methods for diagnosing pulmonary TB [3]. Korean guidelines recommend multiple sputum samples should be taken (at least two and possibly three) [13]. Conventional light microscopy of Ziehl-Neelsen stained smears and fluorescence microscopy are commonly used to diagnose TB, but the sensitivity of smear microscopy is low. On the other hand, fluorescence microscopy is more sensitive than Ziehl-Neelsen staining and takes less time, but has high associated maintenance costs [4]. Recently, light emitting diodes microscopy was introduced and reported to be more sensitive than conventional methods, and the World Health Organization (WHO) has endorsed its use in as an alternative to conventional Ziehl-Neelsen staining [14]. Besides the problem of low sensitivity, sputum smear microscopy is limited by its inability to differentiate $M$. tuberculosis and nontuberculous mycobacteria (NTM). Because the prevalence of NTM lung disease is increasing in many countries including South Korea [15-18], clinicians should be aware of the possibility of NTM as well as M. tuberculosis when confronted with positive acid-fast staining results.

\section{Mycobacterial culture}

Culture of M. tuberculosis is used to confirm a diagnosis of TB and is also needed for drug susceptibility testing (DST) [3]. Mycobacterial culture is much more sensitive than sputum smear, but mycobacterial culture on solid medium requires 4 to 8 weeks for interpretation. Korean researchers have reported when solid culture medium is used, median time from initiation of anti-TB treatment to receipt of mycobacterial culture results by clinicians was 37 days and that median time from treatment initiation to confirmation of DST by requesting clinicians was as much as 80.5 days [19]. This observation indicates clinicians received DST results more than 2 months after initiating treatment.

Mycobacterial culture on liquid medium is significantly faster (10 to 14 days) than that on solid medium. Automatic detection tools, such as the Bactec Mycobacterial Growth Indicator Tube 960 (MGIT 960, Becton-Dickinson, Sparks, MD, USA) or BacT/ALERT (bioMérieux S.A., Marcy l'Etoile, France) have been used, but they need a stable electricity supply, technical support, and expensive reagents. Cross-contamination of M. tuberculosis by NTM or other bacteria is another potential problem. Contamination rates by other bacteria have been reported to be as high as 30\% [20]. Current Korean guidelines recommend that all specimens be cultured on both solid and liquid media to enhance test sensitivity [13].

DSTs that use automated liquid culture system for first line drugs are also available. The Bactec MGIT 960 has been validated to provide susceptibility results for streptomycin, isoniazid, rifampicin, and ethambutol (SIRE) kit and pyrazinamide (PZA) kit. Sensitivity and specificity of this system for detecting rifampin resistance were $99 \%$ to $100 \%$ and $97 \%$ to $100 \%$, respectively, and those for isoniazid resistance were $95 \%$ to $100 \%$ and $100 \%$, respectively. Median turnaround time for the SIRE kit was 5.5 to 8.3 days and for the PZA kit was 5 to 8.2 days [21]. The BacT/ALERT system also provides drug susceptibility results for the same drugs. Its sensitivity and specificity for detecting rifampin resistance were reported to be $99 \%$ to $100 \%$ and $92 \%$ to $100 \%$, respectively, and those for isoniazid resistance were $100 \%$ and $88 \%$ to $100 \%$, respectively. Median turnaround times for the SIRE and PZA kits were 5 to 8.2 and 5 to 7.4 days, respectively [21].

\section{Microscopic-observation drug-susceptibility assay}

The microscopic-observation drug-susceptibility (MODS) assay is an accurate, inexpensive, liquid culture-based diagnostic test that has been endorsed by the WHO for the rapid screening of patients suspected of having multidrug-resistant (MDR)TB [22]. This assay is based on M. tuberculosis culture in Middlebrook $7 \mathrm{H} 9$ liquid broth in four-well columns in a 24-microtiter well plate. The first two wells are drug-free, the third well contains $100 \mu \mathrm{L}$ isoniazid at a concentration of 0.1 or 0.4 $\mu \mathrm{g} / \mathrm{mL}$, and the fourth well contains rifampicin at $1 \mu \mathrm{g} / \mathrm{mL}$. Plates are incubated at $37^{\circ} \mathrm{C}$ and examined using an inverted light microscope at 340 magnification daily from days 4 to 21 , and weekly thereafter until day 40 . Positive MODS cultures are defined by the presence of characteristic cord formation when growth is detected [23].

For rifampicin resistance, pooled estimates for the MODS 
assay were $98.0 \%$ for sensitivity and $99.4 \%$ (range, $95.7 \%$ to 99.9\%) for specificity. For isoniazid resistance with a $0.1 \mu \mathrm{g} / \mathrm{mL}$ cutoff, pooled sensitivity was $97.7 \%$ (range, $94.4 \%$ to $99.1 \%$ ) and pooled specificity was $95.8 \%$ (range, $88.1 \%$ to $98.6 \%$ ), but with a $0.4 \mu \mathrm{g} / \mathrm{mL}$ cutoff, sensitivity was decreased to $90.0 \%$ (range, $84.5 \%$ to $93.7 \%$ ) and specificity was increased to $98.6 \%$ (range, $96.9 \%$ to $99.4 \%$ ). Mean turnaround time was 9.9 days (95\% confidence interval, 4.1 to 15.8 days) [24].

Although the MODS assay is inexpensive and provides accurate results, it has not been used widely in South Korea because the procedure involved is labor-intensive.

\section{Molecular methods}

\section{Nucleic acid amplification testing}

Nucleic acid amplification tests (NAATs) are based on molecular techniques and are used to detect $M$. tuberculosis by detecting and amplifying its RNA or DNA. Of the NAATs described, polymerase chain reaction (PCR) is most commonly used. NAATs for the diagnosis of TB are performed using commercial kits as well as "in-house" test protocols developed in a non-commercial laboratory. The pooled sensitivity of com- mercial NAATs, based on the results of 125 studies, was $85 \%$ (range, 36\% to 100\%) and their pooled specificity was 97\% (range, 54\% to 100\%) [25]. The diagnostic accuracies of PCR tests for smear negative pulmonary TB are also acceptable with reported overall sensitivity and specificity of $72 \%$ and $0.96 \%$, respectively [26]. NAATs are also valuable because they can differentiate $M$. tuberculosis from NTM in specimen with a positive acid-fast staining.

\section{The Xpert MTB/RIF assay}

The Xpert MTB/RIF assay (Cepheid, Sunnyvale, CA, USA) is a rapid, automated, and cartridge-based NAAT that can detect TB along with rifampicin resistance directly from sputum within 2 hours of collection [27]. In a recent study, the Xpert MTB/ RIF assay was found to have a sensitivity of $98.2 \%$ in sputum smear-positive TB cases and of $72.5 \%$ in smear-negative TB cases for the detection of M. tuberculosis [28]. Its sensitivity and specificity for rifampicin resistance were $97.6 \%$ and $98.1 \%$, respectively.

A Korean study conducted in a clinical setting, reported a sensitivity and specificity of $79.5 \%$ and $100.0 \%$, respectively,
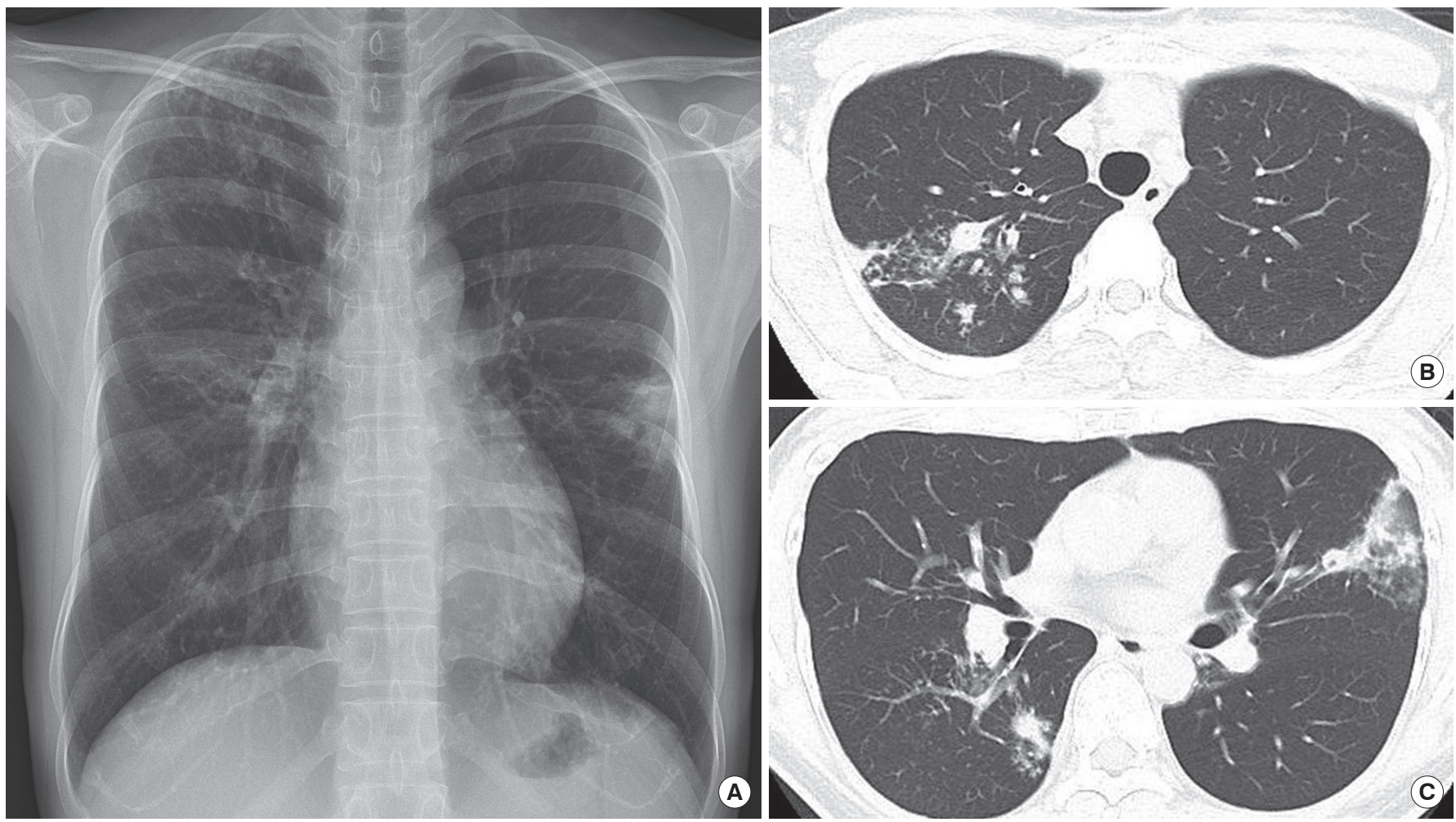

Fig. 2. Pulmonary tuberculosis in a 42-year-old woman with typical radiographic findings. (A) Chest radiograph shows multiple small nodules and patchy consolidation in right upper lung and both middle lung zones. (B, C) Computed tomography scans obtained at levels of trachea and right middle lobar bronchus show cavitary nodules, lobular consolidation, centrilobular nodules and the tree-in-bud pattern in both upper lobes and the superior segment of right lower lobe. 
for the Xpert MTB/RIF assay for a diagnosis of pulmonary TB. For patients with smear positive pulmonary TB, the sensitivity of the Xpert MTB/RIF assay using sputum specimens was $88.9 \%$, and its sensitivity for patients with smear negative TB was $73.1 \%$. The median turnaround time of the assay to the report of results and to confirmation of results by physicians in outpatient settings were 0 day (range, 0 to 1 day) and 6 days (range, 3 to 7 days), respectively [29]. When using bronchoscopic specimens from patients that could not expectorate sputum, the sensitivity and specificity of the Xpert RIF/MTB assay were $81.6 \%$ and $100 \%$, respectively. The sensitivity of this assay is much higher than that of acid fast bacilli smear microscopy, which was reported to be only $13.2 \%$ [30].
The Xpert MTB/RIF assay can also be used to detect rifampin resistance. In a Korean study, the sensitivity and specificity of the assay for detecting rifampin resistance were $100 \%$ and $98.7 \%$, respectively, and its positive and negative predictive values were $86.2 \%$ and $100 \%$, respectively. Furthermore, the positive predictive value of the Xpert MTB/RIF assay for MDRTB diagnosis was found to be $79.3 \%$ [31].

\section{Line probe assay for diagnosis of drug resistance}

Line probe assays (LPAs) provide rapid molecular means for detecting M. tuberculosis and drug resistance. Although LPAs are more complicated and require more time than the Xpert MTB/RIF assay, they provide information on isoniazid and ri-
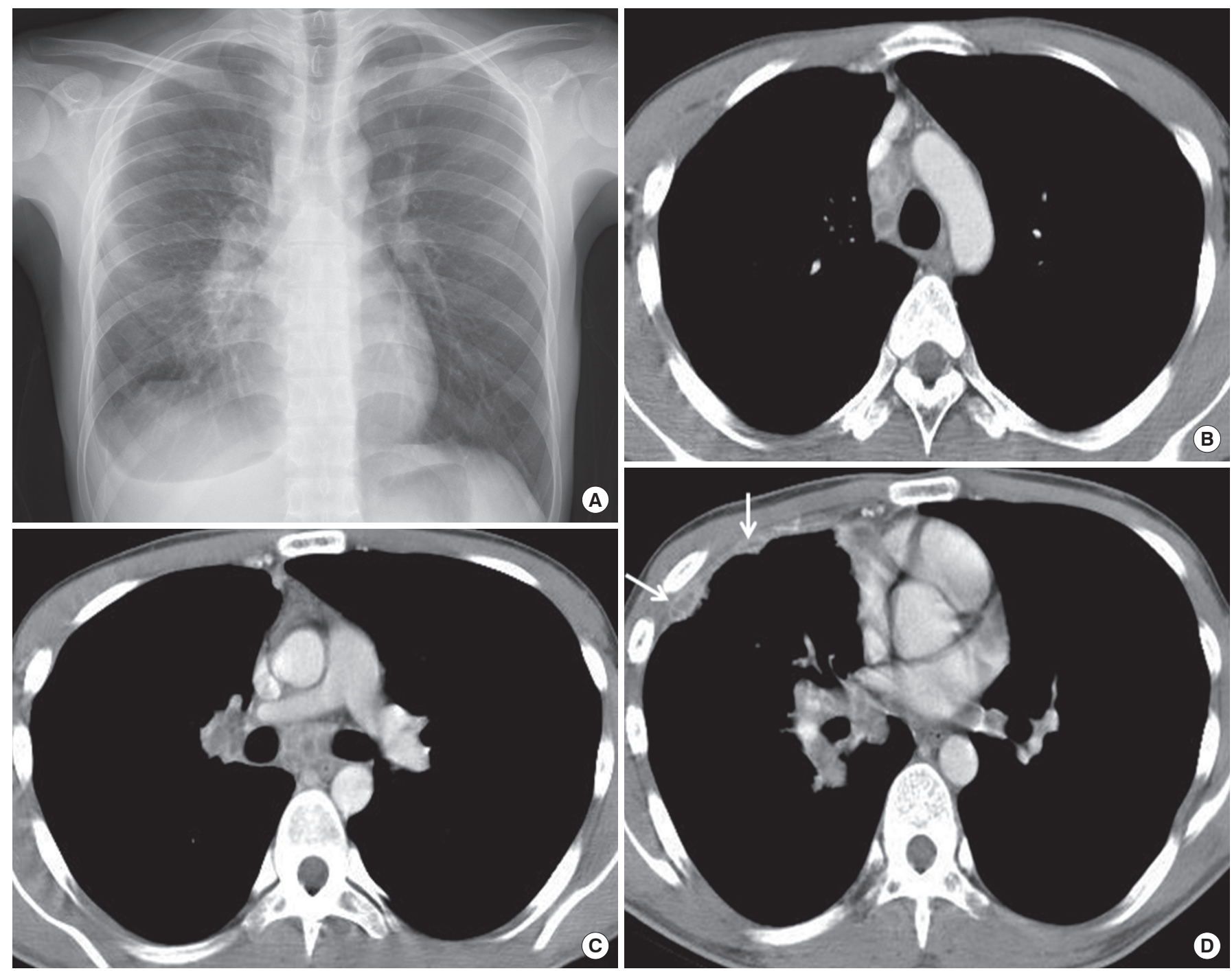

Fig. 3. Tuberculous lymphadenitis and pleurisy in a 28-year-old man with human immunodeficiency virus infection. (A) Chest radiograph shows right hilar enlargement and right pleural effusion. (B, C, D) Serial chest computed tomography images demonstrate right paratracheal, subcarinal, hilar, and peribronchial lymph nodes enlargement with central low attenuation and peripheral rim enhancement. Also note multiple necrotic pleural nodules (arrows) with homogeneous pleural enhancement in the right anterior hemithorax. 
fampicin resistance by identifying mutations in the rpoB, kat $G$, and inhA genes. According to a recent meta-analysis, the pooled sensitivity and specificity for rifampin resistance of LPAs were $96.7 \%$ (range, $95.6 \%$ to $97.5 \%$ ) and $98.8 \%$ (range, $98.2 \%$ to $99.2 \%$ ) and for isoniazid resistance were $90.2 \%$ (range, $88.2 \%$ to $91.9 \%$ ) and $99.2 \%$ (range, $98.7 \%$ to $99.5 \%$ ) [32].

A recent LPA (Genotype MTBDRsl assay, Hain Lifescience $\mathrm{GmbH}$, Nehren, Germany) provides resistance profiles to 2nd line anti-TB drugs. For version 1.0 of the Genotype MTBDRsl assay, pooled sensitivity and specificity for fluoroquinolone resistance were $85.6 \%$ (range, $79.2 \%$ to $90.4 \%$ ) and $98.5 \%$ (range, $95.7 \%$ to $99.5 \%$ ) for $M$. tuberculosis colonies, and $86.2 \%$ (range, $74.6 \%$ to $93.0 \%$ ) and $98.6 \%$ (range, $96.9 \%$ to $99.4 \%$ ) for smear-positive specimens. In addition, pooled sensitivity and specificity for resistance to 2 nd line injectable drugs were $76.5 \%$ (range, $63.3 \%$ to $86.0 \%$ ) and $99.1 \%$ (range, $97.3 \%$ to
99.7\%) for M. tuberculosis colonies, and $87.0 \%$ (range, 38.1\% to $98.6 \%$ ) and $99.5 \%$ (range, $93.6 \%$ to $100.0 \%$ ) for smear-positive specimens [33].

\section{Changes in diagnostic methods for tuberculosis in practice}

Back in the early 2000's, TB treatment was started based on clinical and radiographic suspicion in most South Korean patients. One Korean study reported that diagnosis of TB was confirmed based on clinical and radiographic features in only one-thirds of patients with presumptive TB [34].

However, diagnostic methods for TB that prompt the initiation of TB treatment in practice have changed as new tests have been introduced. Ahn et al. [35] reported a greater proportion of patients were diagnosed with pulmonary TB using NAAT, and that more patients were diagnosed using bron-

Table 1. Characteristic CT findings of pulmonary TB

\begin{tabular}{ll}
\hline Site & \multicolumn{1}{c}{ CT findings } \\
\hline $\begin{array}{l}\text { Parenchyma } \\
\text { Active TB }\end{array}$ & Centrilobular nodules, tree-in-buds pattern, patchy or lobular consolidation, cavity, CT galaxy sign ${ }^{\text {a) }}$, lower lobe consolidation ${ }^{\text {b) }}$ \\
Tuberculoma & Smoothly marginated nodule, no enhancement or ring like enhancement \\
Miliary TB & $1-3$ mm diameter nodule with random distribution, thickening of interlobular septa or intralobular interstitial lines \\
Lymph node & Central areas of low attenuation with peripheral rim enhancement \\
Airway & Circumferential wall thickening and luminal narrowing, with involvement of a long segment of the bronchi \\
Pleura & Pleural effusion with smooth thickening of the visceral and parietal pleural surfaces
\end{tabular}

CT, computed tomography; TB, tuberculosis.

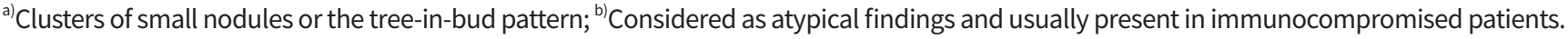

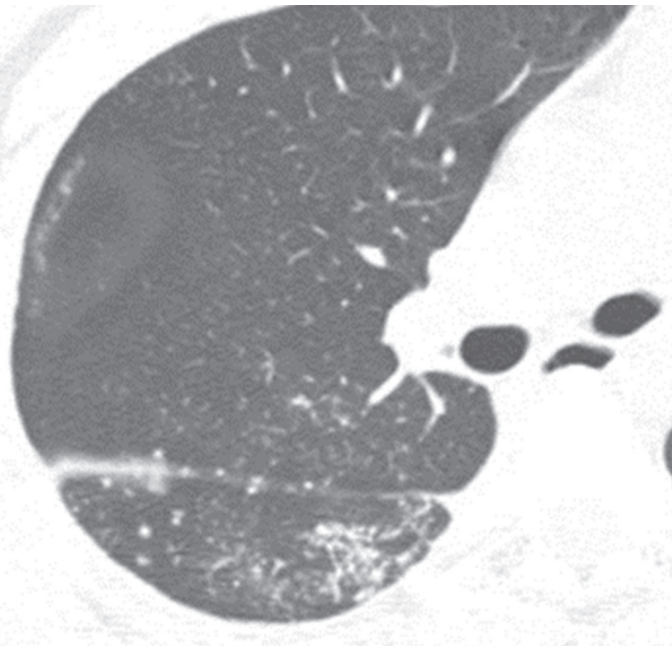

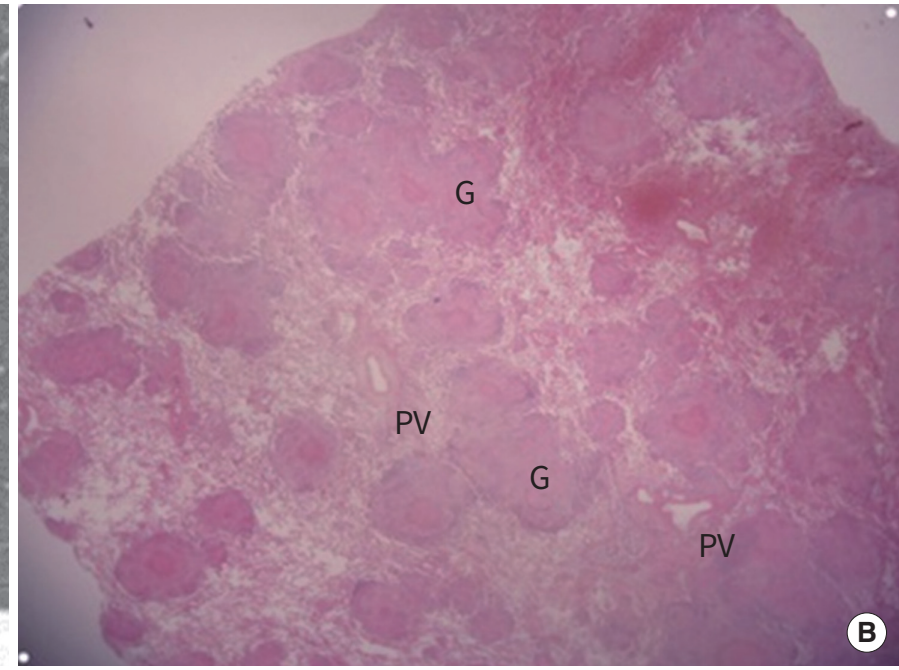

Fig. 4. Miliary tuberculosis in a 21-year-old woman. (A) Chest computed tomography scan obtained at level of right intermediate bronchus shows multiple perifissural nodules and miliary nodules with random distribution in right lung. (B) Photomicrograph of a pathologic specimen obtained by transbronchial lung biopsy discloses numerous granulomas (G) with random distribution (H\&E stain, $\times 40$ ). PV, pulmonary vein. 
choscopic specimens in 2013 than in 2005. Consequently, the proportion of patients clinically diagnosed with pulmonary TB was lower in 2013 than in 2005 [35].

\section{Imaging studies}

\section{Chest radiography}

Chest radiographs play an important role in the screening and diagnosis of pulmonary TB. The typical radiographic find- ings of pulmonary TB in immunocompetent hosts consist of focal or patchy heterogeneous consolidation involving the apical and posterior segments of upper lobes and the superior segments of lower lobes (Fig. 2) [36,37]. Another common finding includes poorly defined nodules and linear opacities. Single or multiple cavities are radiographically evident in $20 \%$ to $45 \%$ of patients $[36,37]$. However, radiographs may be normal or show only mild or nonspecific findings in patients with
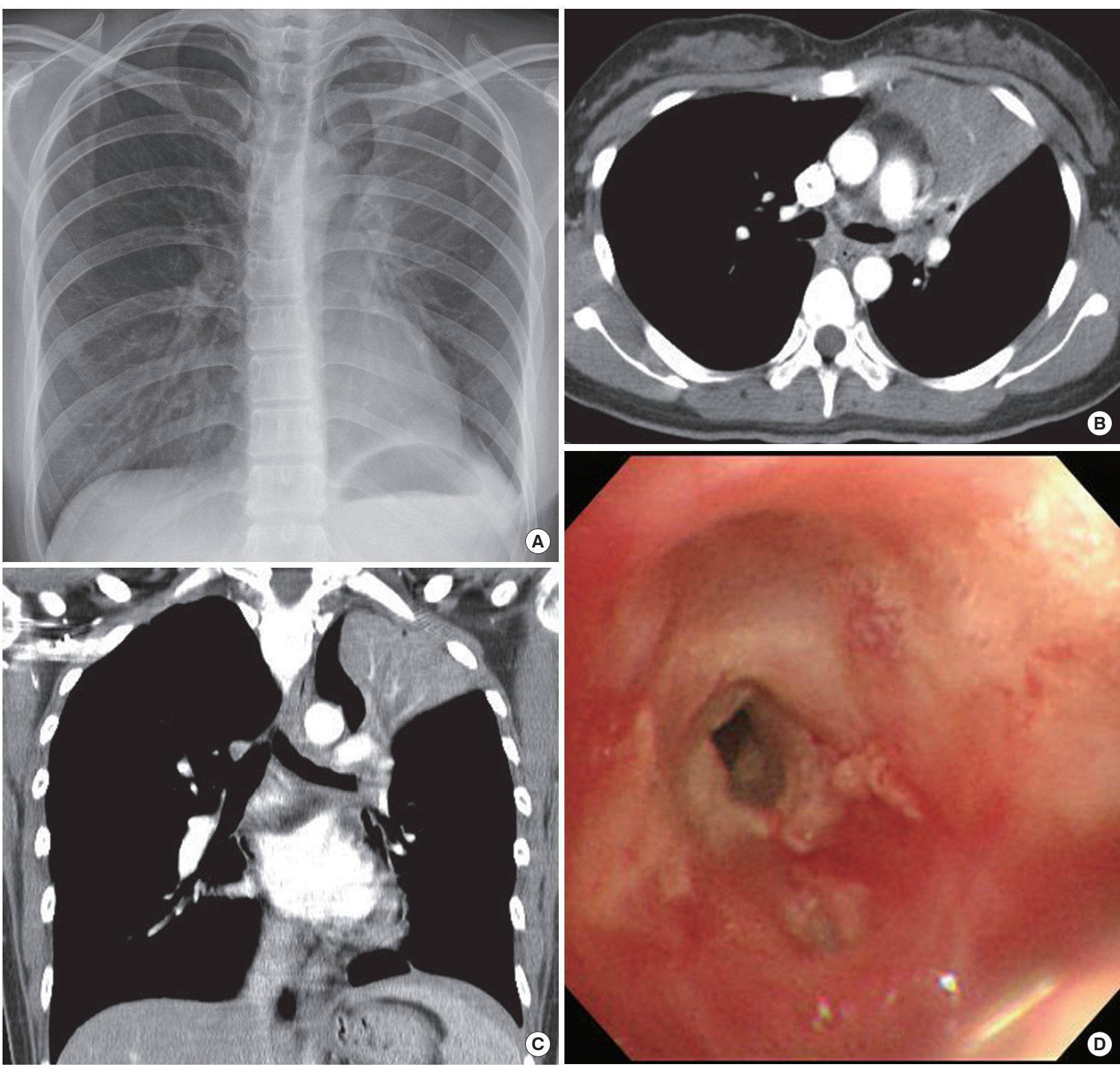

Fig. 5. Bronchial tuberculosis in a 26-year-old woman. (A) Chest radiograph shows left perihilar increased opacity and elevation of left hilum, suggesting left upper lobe atelectasis. (B, C) Axial and coronal reformatted images of chest computed tomography scan show diffuse wall thickening of left main bronchus with left upper lobe atelectasis. (D) Bronchoscopy demonstrates narrowing of the left main bronchus, which was covered with whitish exudates. 
active disease. Actually, initial radiographic diagnoses of TB are correct in only $49 \%$ of cases [38].

In immunocompromised hosts, pulmonary TB manifests as miliary TB, hilar or mediastinal lymphadenopathy, and pleural effusion on chest radiographs (Fig. 3) [10]. Pleural effusion, typically unilateral, occurs in $15 \%$ to $20 \%$ of patients [39]. Although pleural effusion is usually associated with parenchymal abnormalities, it may be the only radiological manifestation of TB.

\section{Computed tomography}

$\mathrm{CT}$ is more sensitive than chest radiography for the detection and characterization of subtle parenchymal disease and mediastinal lymphadenopathy. With CT, a diagnosis of pulmonary TB is correct in $91 \%$ of patients and TB is correctly excluded in $76 \%$ of patients [40]. CT is particularly helpful in the detection of small foci of cavitation in areas of confluent pneumonia and in areas of dense nodularity and scarring [40,41]. For example, in one study of 41 patients with active TB, CT depicted cavities in $58 \%$, whereas chest radiographs showed cavities in only $22 \%$ [42]. CT is also useful for determining disease activity. In one study of 146 TB patients, $80 \%$ with active disease and $89 \%$ with inactive disease were correctly differentiated by high-resolution CT [40]. In addition, CT plays an important role in the detection of pleural or mediastinal complications and in the management of TB by demonstrating sites of cavitation and the extent of active disease [12].
Table 1 summarizes the characteristic $\mathrm{CT}$ findings of parenchymal, lymph node, airway, and pleural TB (Figs. 2-6) [12, 41,43-47].

\section{New imaging modalities}

CT has many advantages for detecting TB lesions and for determining disease activity, but both radiation dose and cost of CT limit its diagnostic use. New imaging modalities, such as, dual-energy subtraction digital radiography (DES-DR) and digital tomosynthesis (DTS) have better diagnostic performances than chest radiography, particularly for the detection of cavity and centrilobular nodules, and reduce radiation hazards associated with repeated CT examinations (Fig. 7). In a comparative study on the diagnostic performances of DES-DR, DTS, and DR, DTS performed better than DES-DR and DR at detecting cavitation (sensitivities of DTS, DES-DR, and DR were $100 \%, 90.3 \%$, and $67.7 \%$, respectively) and nodules (sensitivities were $98.4 \%, 90.2 \%$, and $82 \%$, respectively) [48].

\section{DIAGNOSTIC ALGORITHMS FOR PULMO- NARY TUBERCULOSIS}

Patients suspected of having TB should be referred for medical evaluation. Chest radiographs and multiple sputum samples, for smear microscopy and culture, should be obtained as initial diagnostic steps [13]. In some cases, chest CT scan
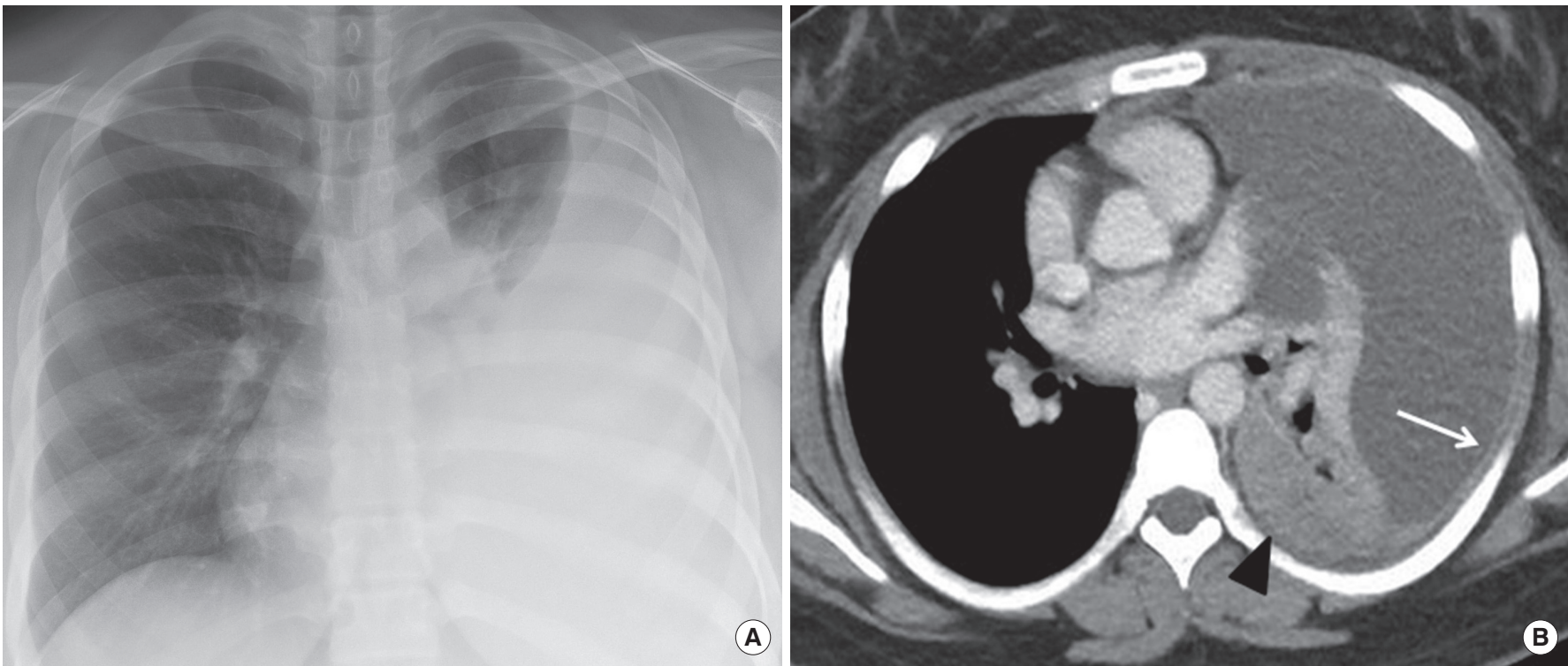

Fig. 6. Tuberculous pleurisy in a 16-year-old girl. (A) Chest radiograph shows left pleural effusion. (B) Chest computed tomography scan obtained at level of left atrium demonstrates left pleural effusion with homogeneous parietal pleural enhancement (arrow). Note the masslike consolidation in the left lower lobe (arrowhead), suggestive of tuberculous pneumonia. 

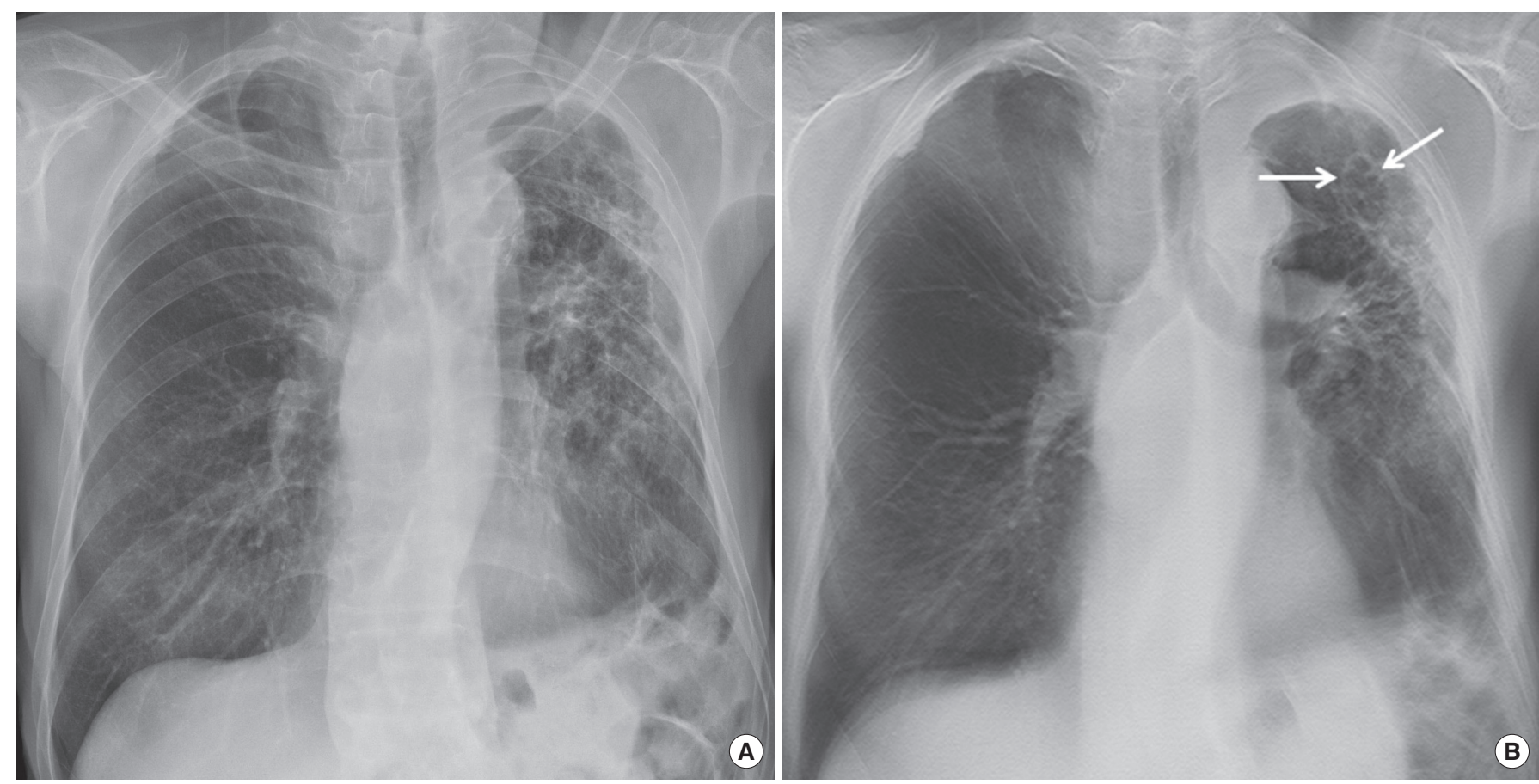

Fig. 7. Multi-drug resistant pulmonary tuberculosis in a 75-year-old man. (A) Chest radiograph shows traction bronchiectasis in the left lung with apical pleural thickening. (B) Chest digital tomosynthesis demonstrate a clearly visible cavitary lesion within the same lesion seen on the chest radiograph (arrows).

aids the identification of a TB lesion or another disease. NAAT can rapidly confirm a TB diagnosis and distinguish M. tuberculosis from non-tuberculous mycobacteria. In particular, the Xpert MTB/RIF assay could provide a rapid diagnosis and determine the presence of rifampicin resistance within 2 hours.

Mycobacterial culture on both solid and liquid media should be performed to confirm diagnoses and to determine drug susceptibilities, and the line-probe assay is useful for detecting resistance to 1 st and 2 nd line anti-TB drugs in patients with a high risk of drug resistance.

\section{CONCLUSION}

TB remains a global health concern. Accurate and rapid diagnosis of TB and the determination of drug resistance using bacterial, molecular, and radiographic evaluations are critical for adequate treatment and for protecting communities.

\section{CONFLICTS OF INTEREST}

No potential conflict of interest relevant to this article was reported.

\section{REFERENCES}

1. World Health Organization. Global tuberculosis report 2016 [Internet]. Geneva: World Health Organization; c2017 [cited 2017 Jun 8]. Available from: http://www.who.int/ tb/publications/global_report/en/.

2. Kim JH, Yim JJ. Achievements in and challenges of tuberculosis control in South Korea. Emerg Infect Dis 2015;21: 1913-20.

3. World Health Organization. Early detection of tuberculosis: an overview of approaches, guidelines and tools [Internet]. Geneva: World Health Organization; c2017 [cited 2017 Jun 8]. Available from: www.who.int/entity/tb/publications/tb-detection-guideline/en/.

4. Steingart KR, Henry M, Ng V, Hopewell PC, Ramsay A, Cunningham J, et al. Fluorescence versus conventional sputum smear microscopy for tuberculosis: a systematic review. Lancet Infect Dis 2006;6:570-81.

5. Ellner JJ. Review: the immune response in human tuberculosis: implications for tuberculosis control. J Infect Dis 1997;176:1351-9.

6. American Thoracic Society. Diagnostic standards and classification of tuberculosis. Am Rev Respir Dis 1990;142:72535. 
7. MacGregor RR. Tuberculosis: from history to current management. Semin Roentgenol 1993;28:101-8.

8. Jones BE, Ryu R, Yang Z, Cave MD, Pogoda JM, Otaya M, et al. Chest radiographic findings in patients with tuberculosis with recent or remote infection. Am J Respir Crit Care Med 1997;156(4 Pt 1):1270-3.

9. Getahun H, Matteelli A, Chaisson RE, Raviglione M. Latent Mycobacterium tuberculosis infection. N Engl J Med 2015; 372:2127-35.

10. Geng E, Kreiswirth B, Burzynski J, Schluger NW. Clinical and radiographic correlates of primary and reactivation tuberculosis: a molecular epidemiology study. JAMA 2005; 293:2740-5.

11. Koh WJ, Jeong YJ, Kwon OJ, Kim HJ, Cho EH, Lew WJ, et al. Chest radiographic findings in primary pulmonary tuberculosis: observations from high school outbreaks. Korean J Radiol 2010;11:612-7.

12. Jeong YJ, Lee KS. Pulmonary tuberculosis: up-to-date imaging and management. AJR Am J Roentgenol 2008;191: 834-44.

13. Joint Committee for the Revision of Korean Guidelines for Tuberculosis, Korea Centers for Disease Control and Prevention. Korean guidelines for tuberculosis. 3rd ed. Cheongju (KR): Korea Centers for Disease Control and Prevention; 2017.

14. World Health Organization. Fluorescent light emitting diode (LED) microscopy for diagnosis of tuberculosis: policy statement [Internet]. Geneva: World Health Organization; c2017 [cited 2017 Jun 8]. Available from: http://www. who.int/laboratory/who_policy_led_microscopy_july10. pdf.

15. Adjemian J, Olivier KN, Seitz AE, Holland SM, Prevots DR. Prevalence of nontuberculous mycobacterial lung disease in U.S. Medicare beneficiaries. Am J Respir Crit Care Med 2012;185:881-6.

16. Moore JE, Kruijshaar ME, Ormerod LP, Drobniewski F, Abubakar I. Increasing reports of non-tuberculous mycobacteria in England, Wales and Northern Ireland, 1995-2006. BMC Public Health 2010;10:612.

17. Chien JY, Lai CC, Sheng WH, Yu CJ, Hsueh PR. Pulmonary infection and colonization with nontuberculous mycobacteria, Taiwan, 2000-2012. Emerg Infect Dis 2014;20: 1382-5.

18. Park YS, Lee CH, Lee SM, Yang SC, Yoo CG, Kim YW, et al. Rapid increase of non-tuberculous mycobacterial lung diseases at a tertiary referral hospital in South Korea. Int J Tuberc Lung Dis 2010;14:1069-71.
19. Joh JS, Lee CH, Lee JE, Park YK, Bai GH, Kim EC, et al. The interval between initiation of anti-tuberculosis treatment in patients with culture-positive pulmonary tuberculosis and receipt of drug-susceptibility test results. J Korean Med Sci 2007;22:26-9.

20. Muyoyeta M, Schaap JA, De Haas P, Mwanza W, Muvwimi MW, Godfrey-Faussett P, et al. Comparison of four culture systems for Mycobacterium tuberculosis in the Zambian National Reference Laboratory. Int J Tuberc Lung Dis 2009; 13:460-5.

21. Piersimoni C, Olivieri A, Benacchio L, Scarparo C. Current perspectives on drug susceptibility testing of Mycobacterium tuberculosis complex: the automated nonradiometric systems. J Clin Microbiol 2006;44:20-8.

22. World Health Organization. Noncommercial culture and drug-susceptibility testing methods for screening patients at risk for multidrug resistant tuberculosis: policy statement [Internet]. Geneva: World Health Organization; c2017 [cited 2017 Jun 8]. Available from: http://whqlibdoc.who. int/publications/2011/9789241501620_eng.pdf.

23. Makamure B, Mhaka J, Makumbirofa S, Mutetwa R, Mupfumi L, Mason P, et al. Microscopic-observation drug-susceptibility assay for the diagnosis of drug-resistant tuberculosis in Harare, Zimbabwe. PLoS One 2013;8:e55872.

24. Minion J, Leung E, Menzies D, Pai M. Microscopic-observation drug susceptibility and thin layer agar assays for the detection of drug resistant tuberculosis: a systematic review and meta-analysis. Lancet Infect Dis 2010;10:68898.

25. Ling DI, Flores LL, Riley LW, Pai M. Commercial nucleic-acid amplification tests for diagnosis of pulmonary tuberculosis in respiratory specimens: meta-analysis and meta-regression. PLoS One 2008;3:e1536.

26. Sarmiento OL, Weigle KA, Alexander J, Weber DJ, Miller WC. Assessment by meta-analysis of PCR for diagnosis of smear-negative pulmonary tuberculosis. J Clin Microbiol 2003;41:3233-40.

27. World Health Organization. Automated real-time nucleic acid amplification technology for rapid and simultaneous detection of tuberculosis and rifampicin resistance: Xpert MTB/RIF assay for the diagnosis of pulmonary and extrapulmonary TB in adults and children: policy update [Internet]. Geneva: World Health Organization; c2017 [cited 2017 Jun 8]. Available from: http://apps.who.int/iris/ handle/10665/112472.

28. Boehme CC, Nabeta P, Hillemann D, Nicol MP, Shenai S, Krapp F, et al. Rapid molecular detection of tuberculosis 


\section{PRECISION AND FUTURE MIEDICINE}

Yeon Joo Jeong, et al.

and rifampin resistance. N Engl J Med 2010;363:1005-15.

29. Kwak N, Choi SM, Lee J, Park YS, Lee CH, Lee SM, et al. Diagnostic accuracy and turnaround time of the Xpert MTB/ RIF assay in routine clinical practice. PLoS One 2013;8: e77456.

30. Lee HY, Seong MW, Park SS, Hwang SS, Lee J, Park YS, et al. Diagnostic accuracy of Xpert(R) MTB/RIF on bronchoscopy specimens in patients with suspected pulmonary tuberculosis. Int J Tuberc Lung Dis 2013;17:917-21.

31. Kim YW, Seong MW, Kim TS, Yoo CG, Kim YW, Han SK, et al. Evaluation of Xpert((R)) MTB/RIF assay: diagnosis and treatment outcomes in rifampicin-resistant tuberculosis. Int J Tuberc Lung Dis 2015;19:1216-21.

32. Nathavitharana RR, Cudahy PG, Schumacher SG, Steingart KR, Pai M, Denkinger CM. Accuracy of line probe assays for the diagnosis of pulmonary and multidrug-resistant tuberculosis: a systematic review and meta-analysis. Eur Respir J 2017;49:1601075.

33. Theron G, Peter J, Richardson M, Warren R, Dheda K, Steingart KR. GenoType(R) MTBDRsl assay for resistance to second-line anti-tuberculosis drugs. Cochrane Database Syst Rev 2016;9:CD010705.

34. Lee CH, Kim WJ, Yoo CG, Kim YW, Han SK, Shim YS, et al. Response to empirical anti-tuberculosis treatment in patients with sputum smear-negative presumptive pulmonary tuberculosis. Respiration 2005;72:369-74.

35. Ahn B, Kim J, Yoo CG, Kim YW, Han SK, Yim JJ. Changes in diagnostic methods for pulmonary tuberculosis between 2005 and 2013. Tuberc Respir Dis (Seoul) 2015;78:227-31.

36. Leung AN. Pulmonary tuberculosis: the essentials. Radiology 1999;210:307-22.

37. Krysl J, Korzeniewska-Kosela M, Muller NL, FitzGerald JM. Radiologic features of pulmonary tuberculosis: an assessment of 188 cases. Can Assoc Radiol J 1994;45:101-7.

38. Cohen JM, Weinreb JC, Redman HC. Postoperative assess- ment of splenorenal shunts with MRI: preliminary investigation. AJR Am J Roentgenol 1986;146:597-600.

39. Epstein DM, Kline LR, Albelda SM, Miller WT. Tuberculous pleural effusions. Chest 1987;91:106-9.

40. Lee KS, Hwang JW, Chung MP, Kim H, Kwon OJ. Utility of $\mathrm{CT}$ in the evaluation of pulmonary tuberculosis in patients without AIDS. Chest 1996;110:977-84.

41. Hatipoglu ON, Osma E, Manisali M, Ucsan ES, Balci P, Akkoclu A, et al. High resolution computed tomographic findings in pulmonary tuberculosis. Thorax 1996;51:397-402.

42. Im JG, Itoh H, Shim YS, Lee JH, Ahn J, Han MC, et al. Pulmonary tuberculosis: CT findings: early active disease and sequential change with antituberculous therapy. Radiology 1993;186:653-60.

43. Murayama S, Murakami J, Hashimoto S, Torii Y, Masuda K. Noncalcified pulmonary tuberculomas: CT enhancement patterns with histological correlation. J Thorac Imaging 1995;10:91-5.

44. McGuinness G, Naidich DP, Jagirdar J, Leitman B, McCauley $\mathrm{DI}$. High resolution $\mathrm{CT}$ findings in miliary lung disease. J Comput Assist Tomogr 1992;16:384-90.

45. Im JG, Song KS, Kang HS, Park JH, Yeon KM, Han MC, et al. Mediastinal tuberculous lymphadenitis: CT manifestations. Radiology 1987;164:115-9.

46. Moon WK, Im JG, Yeon KM, Han MC. Tuberculosis of the central airways: $\mathrm{CT}$ findings of active and fibrotic disease. AJR Am J Roentgenol 1997;169:649-53.

47. Yilmaz MU, Kumcuoglu Z, Utkaner G, Yalniz O, Erkmen G. Computed tomography findings of tuberculous pleurisy. Int J Tuberc Lung Dis 1998;2:164-7.

48. Sharma M, Sandhu MS, Gorsi U, Gupta D, Khandelwal N. Role of digital tomosynthesis and dual energy subtraction digital radiography in detection of parenchymal lesions in active pulmonary tuberculosis. Eur J Radiol 2015; 84:1820-7. 